\title{
Viewpoint \\ Intensity of renal replacement therapy in acute kidney injury: perspective from within the Acute Renal Failure Trial Network Study
}

\author{
Paul M Palevsky ${ }^{1,2}$, Theresa Z O'Connor ${ }^{3}$, Glenn M Chertow ${ }^{4}$, Susan T Crowley ${ }^{3,5}$, \\ Jane Hongyuan Zhang ${ }^{3}$ and John A Kellum ${ }^{2}$ for the US Department of Veterans Affairs/National \\ Institutes of Health Acute Renal Failure Trial Network
}

\author{
${ }^{1}$ Room 7E123 (111F-U), VA Pittsburgh Healthcare System, University Drive, Pittsburgh, PA 15240, USA \\ 2University of Pittsburgh School of Medicine, Pittsburgh, PA 15261, USA \\ 3VA Connecticut Healthcare System, 950 Campbell Avenue, West Haven, CT 06516, USA \\ ${ }^{4}$ Stanford University School of Medicine, Palo Alto, CA 94305, USA \\ ${ }^{5}$ Yale University School of Medicine, New Haven, CT 06520, USA
}

Corresponding author: Paul M Palevsky, Palevsky@pitt.edu

Published: 11 August 2009

This article is online at http://ccforum.com/content/13/4/310

(c) 2009 BioMed Central Ltd
Critical Care 2009, 13:310 (doi:10.1186/cc7901)

survival or recovery of kidney function was found between the two management strategies. Since publication, several aspects of the study design and conduct have been criticized [6-11]. In the present commentary we provide the investigators' perspective on many of the issues that have been raised, the majority of which were carefully considered as the study was designed and conducted [12].

\section{The combined use of intermittent and continuous RRT parallels clinical practice}

We designed the ATN Study as a process-of-care study. As such, the use of both intermittent RRT and continuous RRT was intended to parallel usual clinical practice, in which hemodynamically unstable patients are commonly managed using continuous renal replacement therapy (CRRT) and hemodynamically stable patients are generally treated using intermittent hemodialysis (IHD) [13]. Approximately 40\% of the study participants received both modalities over the course of their illness as their hemodynamic status changed (Table 1). To have restricted patients into a single modality or to have excluded individuals in which more than one modality was used, especially given that study therapy was provided for as long as $\mathbf{2 8}$ days, would have severely undermined the generalizability of the study results. To assure comparable management in both treatment arms, however, conversion between modalities was protocolized - which may have resulted in small differences as compared with clinical practice.

AKI = acute kidney injury; ATN = Acute Renal Failure Trial Network; CRRT = continuous renal replacement therapy; IHD = intermittent hemodialysis; RRT $=$ renal replacement therapy. 


\begin{tabular}{|c|c|c|c|c|c|}
\hline \multirow[b]{2}{*}{$\begin{array}{l}\text { Initial modality } \\
\text { of RRT }\end{array}$} & \multirow[b]{2}{*}{$\begin{array}{c}\text { Number of } \\
\text { modality switches }\end{array}$} & \multicolumn{2}{|c|}{$\begin{array}{l}\text { Intensive management } \\
\text { strategy }(n=563)\end{array}$} & \multicolumn{2}{|c|}{$\begin{array}{l}\text { Less-intensive management } \\
\text { strategy }(n=561)\end{array}$} \\
\hline & & Frequency $^{\mathrm{a}}$ & $\begin{array}{l}\text { Mortality by } \\
\text { day } 60^{\mathrm{b}}\end{array}$ & Frequency $^{\mathrm{a}}$ & $\begin{array}{c}\text { Mortality by } \\
\text { day } 60^{\mathrm{b}}\end{array}$ \\
\hline \multirow[t]{3}{*}{ IHD } & None & $108(19.2)$ & $33(30.6)$ & $138(24.6)$ & $39(28.3)$ \\
\hline & 1 & $18(3.2)$ & $16(88.9)$ & $8(1.4)$ & 7 (87.5) \\
\hline & $\geq 2$ & $27(4.8)$ & $16(59.3)$ & $14(2.5)$ & $4(28.6)$ \\
\hline \multirow[t]{3}{*}{ CRRT/SLED } & None & $203(36.1)$ & 165 (81.3) & $212(37.8)$ & 166 (78.3) \\
\hline & 1 & $136(24.2)$ & 33 (24.3) & $127(22.6)$ & $40(31.5)$ \\
\hline & $\geq 2$ & 58 (10.3) & $31(53.4)$ & $47(8.4)$ & $23(48.9)$ \\
\hline
\end{tabular}

Data presented as $n$ (\%). IHD, intermittent hemodialysis; CRRT/SLED, continuous renal replacement therapy or sustained low-efficiency dialysis. ${ }^{a}$ Calculated as the percentage of participants in the treatment arm. ${ }^{b}$ Calculated as the percentage of participants in the treatment arm treated with a specified initial modality of renal replacement therapy (RRT) and the number of switches in treatment modality.

It has been suggested that the application of continuous and intermittent therapies in the same protocol precludes a valid interpretation of the ATN Study results. This criticism is based on the contention that the dose of intermittent therapy provided in the intensive arm was actually less than the dose of continuous therapy provided in the less-intensive arm [6]. This argument is predicated on one of several mathematical models proposed to establish equivalence of solute clearance when RRT is provided on different schedules [14-17]. Unfortunately, none of these models has been validated in clinical practice, particularly in the acute setting [18].

In designing the protocol, we recognized that combining continuous and intermittent modalities into a single treatment strategy would raise issues regarding the comparability of dose [12]. Given the absence of a reliable model for equivalence of therapies provided on different schedules, we selected doses of IHD and CRRT for the less-intensive treatment arm based on assessment of clinical practice: IHD generally being provided on a thrice-weekly or alternate-day schedule, and CRRT being provided at effluent flow rates of $20 \mathrm{ml} / \mathrm{kg}$ per hour or less [13]. In the intensive treatment arm, we set the dosing of IHD by doubling the frequency of treatment from three to six times per week and we increased the dose of CRRT slightly less than twofold, as previously published data from Ronco and colleagues showed no further improvement in outcomes with doses of CRRT beyond $35 \mathrm{ml} / \mathrm{kg}$ per hour [19].

An alternative (and less controversial) method for assessing equivalence of the treatment dose is to compare the timeaveraged concentration of urea during each of the treatment modalities. While the study was not designed based on this approach, it is notable that the time-averaged blood urea nitrogen concentrations during IHD were remarkably similar to the mean daily concentration during CRRT in both treatment arms: $33 \pm 17 \mathrm{mg} / \mathrm{dl}(12 \pm 6 \mathrm{mmol} / \mathrm{l})$ versus $33 \pm 18 \mathrm{mg} / \mathrm{dl}$ $(12 \pm 6 \mathrm{mmol} / \mathrm{l})$ in the intensive arm, and $48 \pm 19 \mathrm{mg} / \mathrm{dl}$ $(17 \pm 7 \mathrm{mmol} / \mathrm{l})$ versus $47 \pm 23 \mathrm{mg} / \mathrm{dl}(17 \pm 8 \mathrm{mmol} / \mathrm{l})$ in the less-intensive treatment arm [5].

Finally, although the study was not designed to permit rigorous analysis of outcomes by treatment modality, in a post hoc analysis we examined 60-day all-cause mortality between treatment arms as a function of the percentage of time treated with IHD (Figure 1). Following the study protocol, the percentage of time eligible for treatment using IHD was a surrogate for hemodynamic stability. It is therefore not surprising that as the percentage of time eligible for IHD increased, the overall mortality was lower - ranging from more than $80 \%$ in persons with little to no time on IHD, to less than 30\% among those who were treated predominantly with IHD. Similarly, as would be expected given that changes in the modality of RRT within each treatment arm were driven by hemodynamic status, participants who began treatment with intermittent therapy and were switched to CRRT had higher mortality than those who were converted from continuous therapy to IHD (Table 1). Regardless of the subgroup examined, there were no differences in survival as a function of the intensity of RRT.

\section{The initiation of RRT was timely}

Several commentaries have criticized the ATN Study for an unusually long interval between intensive care unit admission and initiation of RRT $[6,10]$. This criticism is based on a misconception regarding the relationship between onset of $\mathrm{AKI}$ and intensive care unit admission. Admission to the intensive care unit cannot be used as a surrogate for the timing of kidney injury. While the interval between intensive care unit admission and initiation of RRT was $6.7 \pm 9.0$ days, the interval between the clinically assessed onset of $A K I$ and study randomization was only $3.2 \pm 2.0$ days. As there is no 


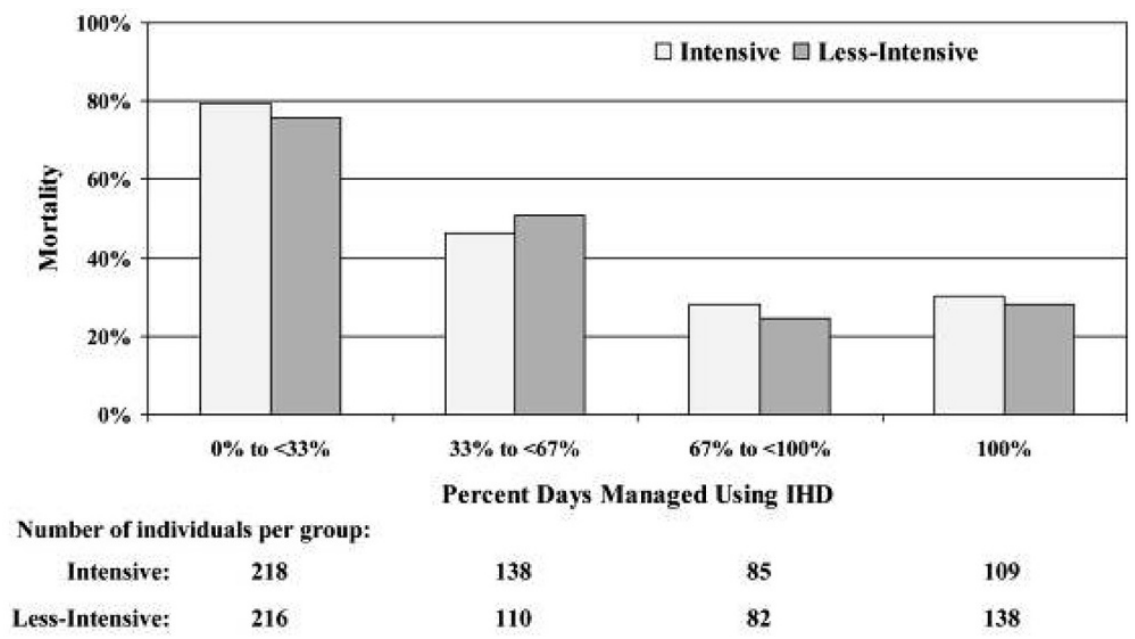

All-cause mortality at 60 days as a function of days managed using intermittent hemodialysis. The time in the intermittent hemodialysis (IHD) phase was defined as the number of days from the first IHD treatment or from the first day after continuous renal replacement therapy (CRRT) or sustained low-efficiency dialysis (SLED) was discontinued until the last day of IHD treatment, the last day before initiation of CRRT or SLED, or the discontinuation of study therapy. Days with IHD and with either CRRT or SLED were counted as in the IHD phase. The percentage of days managed using IHD was calculated by dividing the number of days in the IHD phase by the total number of days of study therapy.

consensus in clinical practice regarding the optimal timing of $\mathrm{RRT}$ in AKI, we left the decision to start RRT to the treating bedside clinical team. Furthermore, the mean blood urea nitrogen at initiation of RRT was lower than that reported in other recent studies [20-22] and was not different between the two treatment arms. We therefore believe that the issue of timing of therapy has little or no impact on the generalizability of the study's results.

The permitted provision of up to 24 hours of CRRT or one IHD session prior to randomization has also been the subject of criticism $[6,10]$. We allowed this limited duration of prerandomization RRT for ethical and safety reasons. As is common in the critical care setting, more than $90 \%$ of enrolled subjects lacked decision-making capacity at enrollment and therefore consent prior to participation had to be obtained from family or other surrogate decision-makers [23]. Since these surrogates were often not available within the hospital, allowing up to 24 hours of nonstudy RRT ensured that the enrollment process did not interfere with appropriate clinical care and delay the initiation of RRT. Although we felt that this brief period of nonstudy RRT would have little impact on study outcomes, we carefully monitored the provision of pre-randomization RRT, collected complete data on these treatments, and evaluated the impact of prerandomization treatment on study outcomes. There were no differences in the use of pre-randomization RRT in the two treatment arms (Table 2). The use of pre-randomization RRT was not associated with 60-day all-cause mortality within the entire cohort (odds ratio $=1.04 ; 95 \%$ confidence interval $=$
0.79 to $1.36 ; P=0.31$ ) and there was no interaction between the use of pre-randomization RRT and the treatment group $(P=0.59)$.

\section{Convective or diffusive solute clearance?}

The ATN Study design has also been criticized for an inadequate use of convective clearance during CRRT [6]. We believe that this criticism is not supported by rigorous evidence. While convective therapies provide greater clearance of higher molecular weight solutes, clearances of lower molecular weight solutes are similar when diffusive and convective therapies are provided at the same flow rates [24]. Furthermore, there is no evidence to support a benefit of convective therapy as compared with diffusive therapy in AKI [25], and one prior study demonstrated that the addition of diffusive clearance to a fixed dose of convection was associated with improved survival [20].

\section{Volume management was similar in the two management strategies}

Although intensity of therapy was defined in terms of low molecular weight solute clearance, the importance of volume removal was explicitly recognized in the study design. During the study, volume management remained under the control of the bedside clinical team. The impact of the study protocol on volume management should have been minimal during CRRT since volume management is independent of solute clearance during continuous therapy. In contrast, we were concerned that when intermittent therapies were employed, restricting the treatment frequency to an every-other-day schedule in the 
Table 2

\begin{tabular}{|c|c|c|c|}
\hline & $\begin{array}{l}\text { Intensive management } \\
\text { strategy }(n=563)\end{array}$ & $\begin{array}{l}\text { Less-intensive management } \\
\text { strategy }(n=561)\end{array}$ & $\begin{array}{c}\text { Odds ratio }(95 \% \mathrm{Cl})^{\mathrm{a}} \\
\text { (between management } \\
\text { strategies) }\end{array}$ \\
\hline Without pre-randomization RRT & $113 / 205(55.1)$ & $106 / 194(54.6)$ & $\begin{array}{c}1.15 \text { (0.85 to } 1.53) \\
P=0.36\end{array}$ \\
\hline With pre-randomization RRT & $189 / 358(52.8)$ & $182 / 366(49.7)$ & $\begin{array}{c}1.00(0.66 \text { to } 1.51) \\
P>0.99\end{array}$ \\
\hline $\begin{array}{l}\text { Odds ratio }(95 \% \mathrm{Cl})^{\mathrm{a}} \\
\text { (within management strategy) }\end{array}$ & $\begin{array}{c}0.90(0.62 \text { to } 1.30) \\
P=0.58\end{array}$ & $\begin{array}{c}1.04(0.71 \text { to } 7.50) \\
P=0.85\end{array}$ & \\
\hline
\end{tabular}

Data presented as number died/number at risk (\%) or odds ratio (95\% confidence interval (CI)). RRT, renal replacement therapy. aOdds ratio calculated by conditional logistic regression modeling adjusted for randomization strata.

less-intensive arm could adversely influence volume management. We therefore allowed the use of isolated ultrafiltration on nondialysis days as required for volume management.

The use of ultrafiltration did not constitute a protocol deviation, as some have contended [6,10], and complete data on these treatments were collected. As expected, more ultrafiltration treatments were provided in the less-intensive management strategy, but even in this study arm there were fewer than 0.5 ultrafiltration treatments per participant during the course of RRT. More importantly, there were no differences in overall fluid balance between the two treatment arms. Over the first 14 days of study therapy, fluid balance was positive by a median of 1.9 I (interquartile range $=-4.8$ to $9.2 \mathrm{I}$ ) in the intensive arm as compared with $1.7 \mathrm{I}$ (interquartile range $=-4.0$ to $8.8 \mathrm{I})$ in the less intensive arm $(P=0.94)$.

\section{Documentation of treatment-associated hypotension}

Critiques have intimated that the frequency of hypotension we reported was unusually high [6]. We previously reported hypotension based on standardized reporting of hypotensionassociated adverse events, including discontinuation of treatment, initiation of vasopressor therapy and any other intervention in response to intradialytic hypotension during each IHD treatment [5]. We also, however, collected predialysis and lowest (nadir) intradialytic blood pressures during each IHD session [5]. Using these data, the frequency of dialysis-associated hypotension in the ATN Study was actually similar to or lower than that reported in previously published trials. Using the same definition as in the French Hemodiafe Study, intradialytic hypotension occurred in $38.3 \%$ of ATN Study participants randomized to the intensive arm and in $36.8 \%$ of patients randomized to the less-intensive arm, as compared with $39 \%$ of the Hemodiafe IHD cohort [22]. Similarly, the requirement for initiation or escalation of vasopressor support in the ATN Study was lower than in a similar cohort described by Schortgen and colleagues [26].
Since changes in hemodynamic stability during continuous therapy were reflected by changes in vasopressor dose, we did not collect similar blood pressure data during CRRT. We observed escalations in vasopressor therapy sufficient to increase the cardiovascular component of the Sequential Organ Failure Assessment score during CRRT in $20.8 \%$ of participants on $3.8 \%$ of treatment days. These data suggest that hypotension is also a frequent occurrence during continuous therapy. While demonstrating that the rates of dialysis-associated hypotension in the ATN Study were not unusually high, these data also suggest that improved strategies are required to minimize hemodynamic instability during both IHD and CRRT.

Commentators have also questioned the difference in the rates of discontinuation of IHD and CRRT as a result of severe hypotension [6]. Once again these differences were intrinsically related to the relationship between hemodynamic status and treatment modality in the study design. The modality of RRT was changed after $35.3 \%$ of IHD treatments that were discontinued due to severe intradialytic hypotension, while RRT was permanently discontinued after only $11.8 \%$ of such episodes. In contrast, in no participants was the modality of RRT changed when CRRT was interrupted due to severe hypotension although the severe hypotension led to permanent discontinuation RRT after $42.3 \%$ of such episodes. Patient outcomes were also strikingly different; $53.8 \%$ of participants died or had life support withdrawn within 1 day of suspension of CRRT due to severe hypotension, as compared with only $12.8 \%$ following discontinuation of an IHD treatment because of severe intradialytic hypotension $(P<0.0001)$.

\section{Defining recovery of kidney function}

Several critiques of the ATN Study have speculated on the low rate of recovery of kidney function $[6,11]$. Unlike other studies that defined recovery of kidney function based on dialysis independence at hospital discharge, we used a more stringent criterion - a measured creatinine clearance 
$>20 \mathrm{ml} /$ minute by day 28 . Using this definition, $41.2 \%$ of study participants in the intensive treatment arm alive at day 28 had recovered kidney function, as did $45.6 \%$ of patients in the less-intensive arm $(P=0.27)$. A substantial number of participants, however, achieved dialysis independence but did not meet the specified definition of recovery of kidney function.

Of the participants alive at day 28,51.5\% and 58.0\% in the intensive and less-intensive strategies were dialysis independent $(P=0.10)$. These percentages increased to $74.6 \%$ and $76.2 \%(P=0.67)$, respectively, among participants alive at day 60 [27]. Although some studies have reported recovery of kidney function in more than $90 \%$ of patients $[19,22]$, the recovery rates we observed were comparable with those seen in other studies $[20,28]$. In the BEST Kidney Study - a prospective observational study of more than 1,000 critically ill patients with $\mathrm{AKI}$ requiring RRT - only $31.3 \%$ of patients were alive off dialysis at hospital discharge [29], as compared with $35.4 \%$ at day 60 in the ATN Study.

\section{Erroneous suggestion of inconsistencies in reported data}

Some authors have even questioned the reliability of our reported data with regard to the delivered dose of therapy, suggesting inconsistencies between the reported mean daily effluent volume during continuous therapy and the values they calculated from the mean daily duration of treatment and the mean values for dialysate, replacement fluid and net ultrafiltration rates [6]. This apparent inconsistency is actually the result of a repeated mathematical error: the product of mean values does not equal the mean of individual products.

$$
\left[\left(\Sigma a_{i}\right) / n\right] \times\left[\left(\Sigma b_{i}\right) / n\right]=\left(\Sigma a_{i}\right) \times\left(\Sigma b_{i}\right) / n^{2} \neq\left[\Sigma\left(a_{i} \times b_{i}\right)\right] / n
$$

It is therefore not surprising that the values these authors have attempted to calculate do not correspond to the actual measured values.

\section{Conclusions}

We designed the US Department of Veterans Affairs/National Institutes of Health ATN Study to test the hypothesis that more intensive RRT in critically ill patients with AKI is associated with improved outcomes. The study results do not support the contention that increasing intensity of therapy beyond a sufficient dose is associated with decreased mortality, improved recovery of kidney function or differences in the course of nonrenal organ failure. That is not to say that the study supports an approach of therapeutic nihilism, as suggested by Ronco and colleagues [6]. Rather, since the less-intensive strategy provided a level of renal support that often exceeds typical clinical practice, our results suggest there needs to be a greater emphasis on ensuring that an appropriate prescribed dose of therapy is actually delivered. For patients receiving intermittent therapy, this will require monitoring the delivered dose, with careful attention to ensure delivery of $K t / V_{\text {urea }}$ of at least 1.2 per treatment. For patients receiving continuous therapy, emphasis needs to be on ensuring that treatment times are maximized, since prior studies have suggested substantial underestimation of interruptions of treatment [30].

While it has been suggested that the use of a fixed dosed of therapy throughout the dynamic course of an episode of AKI may not be appropriate [9], we believe this hypothesis is untested and requires rigorous evaluation. We agree that treatment needs to be individualized and that more intensive therapy may be required in some situations. Although our study design used protocol-based criteria to guide switching between modalities of therapy, it also needs to be recognized that these criteria were empirically derived, using expert opinion and consensus, and remain untested as to whether they represent the best approach for every patient. For all modalities, new strategies to minimize complications of therapy - including hypotension and electrolyte disturbances need to be implemented. In addition, the optimal timing of RRT and fluid management during therapy need to be rigorously evaluated.

While we need to optimize the care delivered, the results of the ATN Study also suggest that merely modifying the prescription and delivery of RRT is unlikely to result in substantial improvement in outcomes. We must recognize the limits of the treatment and shift our focus to other strategies for prevention and treatment of AKI.

\section{Competing interests}

The authors declare that they have no competing interests.

\section{Acknowledgements}

Supported by the Cooperative Studies Program of the Department of Veterans Affairs Office of Research and Development and by the National Institute of Diabetes and Digestive and Kidney Diseases (interagency agreement Y1-DK-3508-01).

\section{References}

1. Davenport A, Bouman C, Kirpalani A, Skippen P, Tolwani A, Mehta RL, Palevsky PM: Delivery of renal replacement therapy in acute kidney injury: what are the key issues? Clin J Am Soc Nephrol 2008, 3:869-875.

2. Kellum JA, Mehta RL, Angus DC, Palevsky P, Ronco C: The first international consensus conference on continuous renal replacement therapy. Kidney Int 2002, 62:1855-1863.

3. Palevsky PM: Clinical review: timing and dose of continuous renal replacement therapy in acute kidney injury. Crit Care 2007, 11:232.

4. Ronco C, Ricci Z, Bellomo R: Current worldwide practice of dialysis dose prescription in acute renal failure. Curr Opin Crit Care 2006, 12:551-556.

5. Palevsky PM, Zhang JH, O'Connor TZ, Chertow GM, Crowley ST, Choudhury D, Finkel K, Kellum JA, Paganini E, Schein RM, Smith MW, Swanson KM, Thompson BT, Vijayan A, Watnick S, Star RA, Peduzzi $P$ for the VA/NIH Acute Renal Failure Trial Network: Intensity of renal support in critically ill patients with acute kidney injury. N Engl J Med 2008, 359:7-20.

6. Ronco C, Cruz D, van Straaten HO, Honore P, House A, Bin D, Gibney N: Dialysis dose in acute kidney injury: no time for therapeutic nihilism -a critical appraisal of the Acute Renal Failure Trial Network study. Crit Care 2008, 12:308. 
7. Ronco $\mathrm{C}$, Honore $\mathrm{P}$ : Renal support in critically ill patients with acute kidney injury [letter]. N Engl J Med 2008, 359:1959; author reply 1961-1962.

8. Bouchard J, Macedo E, Mehta RL: Renal support in critically ill patients with acute kidney injury [letter]. N Engl J Med 2008, 359:1959-1960; author reply 1961-1962.

9. Maynar-Moliner J, Sanchez-lzquierdo-Riera JA, Herrera-Gutierrez M: Renal support in critically ill patients with acute kidney injury [letter]. N Engl J Med 2008, 359:1960; author reply 19611962.

10. Bagshaw SM, Gibney N: Renal support in critically ill patients with acute kidney injury [letter]. N Engl J Med 2008, 359:19601961; author reply 1961-1962.

11. Uchino S, Bell M, Bellomo R: Renal support in critically ill patients with acute kidney injury [letter]. N Engl J Med 2008, 359:1961; author reply 1961-1962.

12. Palevsky PM, O'Connor T, Zhang JH, Star RA, Smith MW: Design of the VA/NIH Acute Renal Failure Trial Network (ATN) Study: intensive versus conventional renal support in acute renal failure. Clin Trials 2005, 2:423-435.

13. Overberger $P$, Pesacreta M, Palevsky PM: Management of renal replacement therapy in acute kidney injury: a survey of practitioner prescribing practices. Clin J Am Soc Nephrol 2007, 2: 623-630.

14. Keshaviah PR, Nolph KD, Van Stone JC: The peak concentration hypothesis: a urea kinetic approach to comparing the adequacy of continuous ambulatory peritoneal dialysis (CAPD) and hemodialysis. Perit Dial Int 1989, 9:257-260.

15. Casino FG, Lopez T: The equivalent renal urea clearance: a new parameter to assess dialysis dose. Nephrol Dial Transplant 1996, 11:1574-1581.

16. Clark WR, Mueller BA, Kraus MA, Macias WL: Dialysis prescription and kinetics in acute renal failure. Adv Ren Replace Ther 1997, 4(2 Suppl 1):64-71.

17. Gotch FA: The current place of urea kinetic modelling with respect to different dialysis modalities. Nephrol Dial Transplant 1998, 13(Suppl 6):10-14.

18. Gotch FA, Sargent JA, Keen ML: Whither goest Kt/V? Kidney Int Supp/ 2000, 76:S3-S18.

19. Ronco C, Bellomo R, Homel P, Brendolan A, Dan M, Piccinni P, La Greca G: Effects of different doses in continuous venovenous haemofiltration on outcomes of acute renal failure: a prospective randomised trial. Lancet 2000, 356:26-30.

20. Saudan P, Niederberger M, De Seigneux S, Romand J, Pugin J, Perneger T, Martin PY: Adding a dialysis dose to continuous hemofiltration increases survival in patients with acute renal failure. Kidney Int 2006, 70:1312-1317.

21. Liu KD, Himmelfarb J, Paganini E, Ikizler TA, Soroko SH, Mehta $\mathrm{RL}$, Chertow GM: Timing of initiation of dialysis in critically ill patients with acute kidney injury. Clin J Am Soc Nephrol 2006, 1:915-919.

22. Vinsonneau C, Camus C, Combes A, Costa de Beauregard MA, Klouche K, Boulain T, Pallot JL, Chiche JD, Taupin P, Landais P, Dhainaut J-F, for the Hemodiafe Study Group: Continuous venovenous haemodiafiltration versus intermittent haemodialysis for acute renal failure in patients with multiple-organ dysfunction syndrome: a multicentre randomised trial. Lancet 2006, 368:379-385.

23. Crowley ST, Chertow GM, Vitale J, O'Connor T, Zhang J, Schein RM, Choudhury D, Finkel K, Vijayan A, Paganini E, Palevsky PM, for the VA/NIH Acute Renal Failure Trial Network Study Group: Lessons for successful study enrollment from the Veterans Affairs/National Institutes of Health Acute Renal Failure Trial Network Study. Clin J Am Soc Nephrol 2008, 3:955-961.

24. Troyanov S, Cardinal J, Geadah D, Parent D, Courteau S, Caron $S$, Leblanc M: Solute clearances during continuous venovenous haemofiltration at various ultrafiltration flow rates using Multiflow-100 and HF1000 filters. Nephrol Dial Transplant 2003, 18:961-966.

25. Pannu N, Klarenbach S, Wiebe N, Manns B, Tonelli M: Renal replacement therapy in patients with acute renal failure: a systematic review. JAMA 2008, 299:793-805.

26. Schortgen F, Soubrier N, Delclaux C, Thuong M, Girou E, BrunBuisson C, Lemaire F, Brochard L: Hemodynamic tolerance of intermittent hemodialysis in critically ill patients: usefulness of practice guidelines. Am J Respir Crit Care Med 2000, 162: 197-202.
27. Palevsky PM, Franchini R, O'Connor TZ, Zhang JH: Recovery of kidney function in critically ill patients with acute kidney injury treated with intensive versus less intensive renal replacement therapy [abstract]. J Am Soc Nephrol 2008, 19:790A.

28. Tolwani AJ, Campbell RC, Stofan BS, Lai KR, Oster RA, Wille KM: Standard versus high-dose CVVHDF for ICU-related acute renal failure. J Am Soc Nephrol 2008, 19:1233-1238.

29. Uchino S, Bellomo R, Kellum JA, Morimatsu H, Morgera S, Schetz MR, Tan I, Bouman C, Macedo E, Gibney N, Tolwani A, Oudemans-Van Straaten HM, Ronco C; Beginning and Ending Supportive Therapy for the Kidney (B.E.S.T. Kidney) Investigators Writing Committee: Patient and kidney survival by dialysis modality in critically ill patients with acute kidney injury. Int $J$ Artif Organs 2007, 30:281-292.

30. Venkataraman R, Kellum JA, Palevsky P: Dosing patterns for continuous renal replacement therapy at a large academic medical center in the United States. J Crit Care 2002, 17:246250. 